\title{
SOME RESULTS ON THE $q$-ANALOGUES OF THE INCOMPLETE FIBONACCI AND LUCAS POLYNOMIALS
}

\author{
H. M. SRIVASTAVA, NAIM TUGLU, AND MIRAC ÇETIN
}

Received 27 January, 2019

\begin{abstract}
In the present paper, we introduce new families of the $q$-Fibonacci and $q$-Lucas polynomials, which are represented here as the incomplete $q$-Fibonacci polynomials $F_{n}^{k}(x, s, q)$ and the incomplete $q$-Lucas polynomials $L_{n}^{k}(x, s, q)$, respectively. These polynomials provide the $q$-analogues of the incomplete Fibonacci and Lucas numbers. We give several properties and generating functions of each of these families $q$-polynomials. We also point out the fact that the results for the $q$-analogues which we consider in this article for $0<q<1$ can easily be translated into the corresponding results for the $(p, q)$-analogues (with $0<q<p \leqq 1$ ) by applying some obvious parametric variations, the additional parameter $p$ being redundant.
\end{abstract}

2010 Mathematics Subject Classification: 11B39; 05A30

Keywords: Fibonacci polynomials and numbers, Lucas polynomials and numbers, $q$-Fibonacci polynomials, $q$-Lucas polynomials, incomplete Fibonacci numbers, incomplete Lucas numbers, equivalence of the $q$-analogues and the corresponding $(p, q)$-analogues

\section{INTRODUCTION}

The Fibonacci numbers are defined by the following recurrence relation:

$$
F_{n+1}=F_{n}+F_{n-1} \quad(n \in\{0,1,2, \cdots\})
$$

with the initial conditions $F_{0}=0$ and $F_{1}=1$ and the Lucas numbers are defined by the same recurrence relation with the different initial conditions $L_{0}=2$ and $L_{1}=1$.

In existing literature, there are many extensions and generalizations of the Fibonacci numbers. For instance, Filipponi [13] defined the incomplete Fibonacci and Lucas numbers as follows:

$$
F_{n}(k)=\sum_{j=0}^{k}\left(\begin{array}{c}
n-1-j \\
j
\end{array}\right) \quad\left(0 \leqq k \leqq\left\lfloor\frac{n-1}{2}\right\rfloor\right)
$$

and

$$
L_{n}(k)=\sum_{j=0}^{k} \frac{n}{n-j}\left(\begin{array}{c}
n-j \\
j
\end{array}\right) \quad\left(0 \leqq k \leqq\left\lfloor\frac{n}{2}\right\rfloor\right),
$$


where $n=1,2,3, \cdots$. We note that

$$
F_{n}\left(\left\lfloor\frac{n-1}{2}\right\rfloor\right)=F_{n}
$$

and

$$
L_{n}\left(\left\lfloor\frac{n}{2}\right\rfloor\right)=L_{n} .
$$

The generating functions of these numbers were studied by Pintér and Srivastava [17]. Many other authors have also studied this topic (see, for example, [8-12,18,19, 21,22]).

For $0<q<1$, the $q$-integer is defined by

$$
[n]:=[n]_{q}=\frac{1-q^{n}}{1-q}
$$

and the $q$-factorial is defined by

$$
[n] !:= \begin{cases}{[n] \cdot[n-1] \cdots[1]} & (n=1,2,3, \cdots) \\ 1 & (n=0) .\end{cases}
$$

The $q$-binomial coefficients are defined by

$$
\left[\begin{array}{l}
n \\
k
\end{array}\right]=\frac{[n] !}{[n-k] ![k] !} \quad(0 \leqq k \leqq n)
$$

with (see [2] and [20])

$$
\left[\begin{array}{l}
n \\
0
\end{array}\right]=1 \quad \text { and } \quad\left[\begin{array}{l}
n \\
k
\end{array}\right]=0 \quad(n<k) .
$$

The Heine's binomial formula is recalled here as follows (see [2, p. 2]):

$$
\frac{1}{(1-x)_{q}^{n}}=1+\sum_{j=1}^{\infty} \frac{[n]_{q}[n+1]_{q} \cdots[n+j-1]_{q}}{[j]_{q} !} x^{j} .
$$

The $q$-difference operator $D_{q}$ is defined as follows:

$$
D_{q} f(x)=\frac{f(x)-f(q x)}{(1-q) x}
$$

if $x \neq 0$.

Cigler [6] introduced the $q$-Fibonacci polynomials which are defined below:

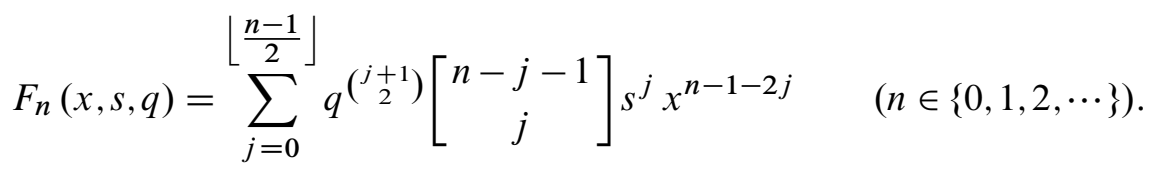


Also, in [4], we have the following explicit formula for the $q$-Lucas polynomials:

$$
L_{n}(x, s, q)=\sum_{j=0}^{\left\lfloor\frac{n}{2}\right\rfloor} q^{\left(\begin{array}{l}
j \\
2
\end{array}\right)} \frac{[n]}{[n-j]}\left[\begin{array}{c}
n-j \\
j
\end{array}\right] s^{j} x^{n-2 j} \quad(n \in\{0,1,2, \cdots\}) .
$$

A recurrence relation for the $q$-Fibonacci polynomials is given by

$$
F_{n}(x, s, q)=x F_{n-1}(x, s, q)+(q-1) s D_{q} F_{n-1}(x, s, q)+s F_{n-2}(x, s, q),
$$

with the initial values $F_{0}(x, s, q)=0$ and $F_{1}(x, s, q)=1$. The $q$-Lucas polynomials satisfy the same recurrence relation as above:

$$
L_{n}(x, s, q)=x L_{n-1}(x, s, q)+(q-1) s D_{q} L_{n-1}(x, s, q)+s L_{n-2}(x, s, q),
$$

but with the initial values given by (see, for details, [4])

$$
L_{0}(x, s, q)=2 \quad \text { and } \quad L_{1}(x, s, q)=x .
$$

The following formulas provide relationship between these polynomials:

$$
L_{n}(x, s, q)=F_{n+1}(x, s, q)+s F_{n-1}(x, s, q)
$$

and

$$
L_{n}(x, q s, q)=F_{n+1}(x, s, q)+q^{n} s F_{n-1}(x, s, q) .
$$

For more details about the $q$-analogues of the Fibonacci polynomials, see [1,3-5, 7, 14]. There are only a few studies for the $q$-Fibonacci and $q$-Lucas polynomials and for the extensions and generalizations of these polynomials.

We choose to remark in passing that several authors (see, for example, [23] and [24]) studied the so-called $(p, q)$-Fibonacci and $(p, q)$-Lucas polynomials by introducing a seemingly redundant parameter $p$, since the so-called $(p, q)$-number $[n]_{p, q}$ is given (for $0<q<p \leqq 1$ ) by

$$
\begin{aligned}
& {[n]_{p, q}:= \begin{cases}\frac{p^{n}-q^{n}}{p-q} & (n \in\{1,2,3, \cdots\}) \\
0 & (n=0)\end{cases} } \\
& =p^{n-1}\left(\frac{q}{p}\right)_{n} \\
& =: p^{n-1}[n]_{\frac{q}{p}}
\end{aligned}
$$

and

$$
\begin{aligned}
{[n]_{q} } & :=\frac{1-q^{n}}{1-q} \\
& =p^{1-n}\left(\frac{p^{n}-(p q)^{n}}{p-(p q)}\right)
\end{aligned}
$$




$$
=p^{1-n}[n]_{p, p q},
$$

which do exhibits the fact that the results for the $q$-analogues which we consider in this article for $0<q<1$ can easily be translated into the corresponding results for the $(p, q)$-analogues (with $0<q<p \leqq 1)$ by applying some obvious parametric variations, the additional parameter $p$ being redundant.

The aim of this paper is to introduce and study $q$-analogues of (1.1) and (1.2). We thus investigate the incomplete $q$-Fibonacci and $q$-Lucas polynomials and derive some of their properties including their generating functions.

\section{MAIN RESULTS}

We define the incomplete $q$-Fibonacci and $q$-Lucas polynomials by using the explicit formulas as follows.

Definition 1. The incomplete $q$-Fibonacci polynomials are defined by

$$
\mathcal{F}_{n}^{k}:=F_{n}^{k}(x, s, q)=\sum_{j=0}^{k} q^{(j+1)}\left[\begin{array}{c}
n-j-1 \\
j
\end{array}\right] s^{j} x^{n-1-2 j}
$$

for $0 \leqq k \leqq\left\lfloor\frac{n-1}{2}\right\rfloor$.

If we take $q \rightarrow 1-$ in (2.1), we get incomplete bivariate Fibonacci numbers in [22]. On the other hand, if we take $q \rightarrow 1-$ and $s=x=1$ in (2.1), we get incomplete Fibonacci numbers studied in [13].

TABLE 1. Incomplete $q$-Fibonacci polynomials for $n=1, \cdots, 8$ and $k=0,1,2,3,4$.

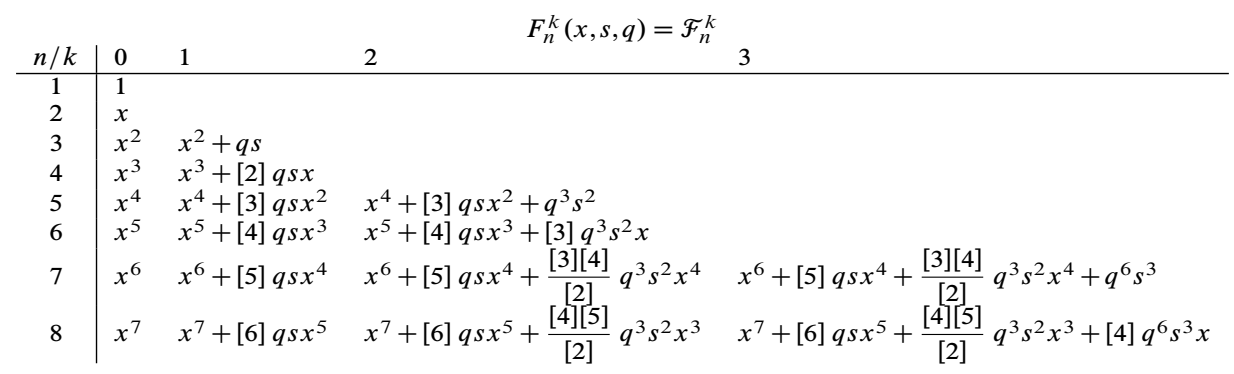

We now define incomplete $q$-Lucas polynomials.

Definition 2. The incomplete $q$-Lucas polynomials are defined by

$$
\mathscr{L}_{n}^{k}:=L_{n}^{k}(x, s, q)=\sum_{j=0}^{k} q^{\left(\begin{array}{c}
j \\
2
\end{array}\right)} \frac{[n]}{[n-j]}\left[\begin{array}{c}
n-j \\
j
\end{array}\right] s^{j} x^{n-2 j}
$$

for $0 \leqq k \leqq\left\lfloor\frac{n}{2}\right\rfloor$. 
TABLE 2. Incomplete $q$-Lucas polynomials for $n=1, \cdots, 7$ and $k=0,1,2,3$.

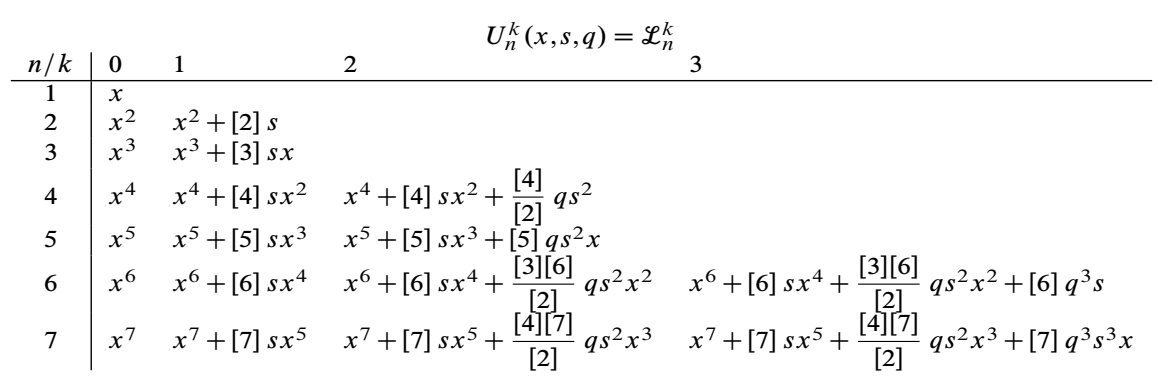

In particular, if we take $q \rightarrow 1-$ in (2.2), then we get incomplete bivariate Lucas numbers studied in [22]. Moreover, if we take $q \rightarrow 1-$ and $s=x=1$ in (2.2), then we get incomplete Lucas numbers studied in [13].

\subsection{Recurrence relations}

Theorem 1. The recurrence relation of the incomplete q-Fibonacci polynomials is given by

$$
\mathscr{F}_{n+2}^{k+1}=x \mathcal{F}_{n+1}^{k+1}+(q-1) s D_{q} \mathcal{F}_{n+1}^{k}+s \mathscr{F}_{n}^{k}
$$

for $0 \leqq k \leqq \frac{n-2}{2}$.

Proof. We find from (2.1) that

$$
\begin{aligned}
& x \mathcal{F}_{n+1}^{k+1}+(q-1) s D_{q} \widetilde{F}_{n+1}^{k}+s \mathscr{F}_{n}^{k} \\
& =\sum_{j=0}^{k+1} q^{\left(\begin{array}{c}
j+1 \\
2
\end{array}\right)}\left[\begin{array}{c}
n-j \\
j
\end{array}\right] s^{j} x^{n+1-2 j} \\
& +(q-1) \sum_{j=0}^{k} q^{\left(\begin{array}{c}
j+1 \\
2
\end{array}\right)}\left[\begin{array}{c}
n-j \\
j
\end{array}\right][n-2 j] s^{j+1} x^{n-1-2 j} \\
& +\sum_{j=0}^{k} q^{\left(\begin{array}{c}
j+1 \\
2
\end{array}\right)}\left[\begin{array}{c}
n-j-1 \\
j
\end{array}\right] s^{j+1} x^{n-1-2 j} \\
& =\sum_{j=0}^{k+1}\left\{q^{\left(\begin{array}{c}
j+1 \\
2
\end{array}\right)}\left[\begin{array}{c}
n-j \\
j
\end{array}\right]+(q-1) q^{\left(\begin{array}{c}
j \\
2
\end{array}\right)}\left[\begin{array}{c}
n-j+1 \\
j-1
\end{array}\right][n-2 j+2]\right. \\
& \left.+q^{\left(\begin{array}{c}
j \\
2
\end{array}\right)}\left[\begin{array}{c}
n-j \\
j-1
\end{array}\right]\right\} s^{j} x^{n+1-2 j} .
\end{aligned}
$$

It is known that (see [5])

$$
q^{\left(\begin{array}{c}
j+1 \\
2
\end{array}\right)}\left[\begin{array}{c}
n-j+1 \\
j
\end{array}\right]=
$$




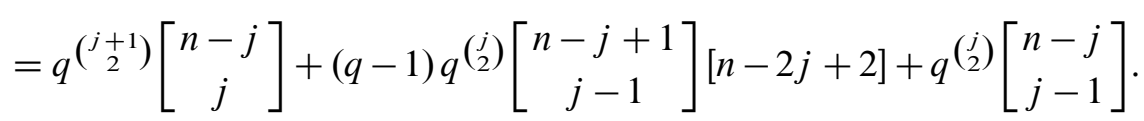

Therefore, the recurrence relation (2.3) is seen to hold true.

In Theorem 1, by taking $q \rightarrow 1-$ and $x=s=1$, we get the following result:

$$
F_{n+2}(k+1)=F_{n+1}(k+1)+F_{n}(k) \quad\left(0 \leqq k \leqq \frac{n-2}{2}\right),
$$

where $F_{n}(k)$ are the incomplete Fibonacci numbers studied in [13].

The following theorem results, in part, from the recurrence relation (2.3).

Theorem 2. The following non-homogeneous recurrence relation of the incomplete q-Fibonacci polynomials holds true:

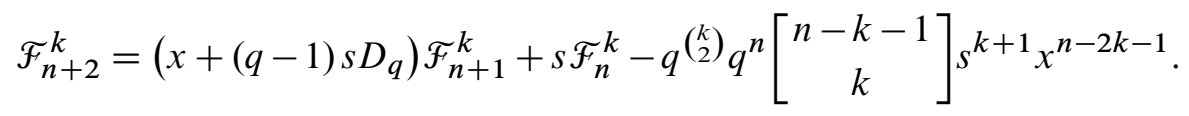

Proof. The proof of the non-homogeneous recurrence relation (2.4) follows from Definition 1 and the equation (2.3).

Theorem 3. It is asserted that

$$
\mathscr{L}_{n}^{k}=\mathscr{F}_{n+1}^{k}+s \mathscr{F}_{n-1}^{k-1} \quad\left(0 \leqq k \leqq \frac{n}{2}\right) .
$$

Proof. Using the equation (2.1), we have

$$
\begin{aligned}
& \mathcal{F}_{n+1}^{k}+s \mathscr{F}_{n-1}^{k-1}=\sum_{j=0}^{k} q^{\left(\begin{array}{c}
j+1 \\
2
\end{array}\right)}\left[\begin{array}{c}
n-j \\
j
\end{array}\right] s^{j} x^{n-2 j}+s \sum_{j=0}^{k-1} q^{\left(\begin{array}{c}
j+1 \\
2
\end{array}\right)}\left[\begin{array}{c}
n-j-2 \\
j
\end{array}\right] s^{j} x^{n-2-2 j} \\
& =\sum_{j=0}^{k} q^{\left(\begin{array}{c}
j \\
2
\end{array}\right)}\left\{q^{-\left(\begin{array}{c}
j \\
2
\end{array}\right)} q^{\left(\begin{array}{c}
j+1 \\
2
\end{array}\right)}\left[\begin{array}{c}
n-j \\
j
\end{array}\right]+\left[\begin{array}{c}
n-j-1 \\
j-1
\end{array}\right]\right\} s^{j} x^{n-2 j} \\
& =\sum_{j=0}^{k} q^{\left(\begin{array}{c}
j \\
2
\end{array}\right)}\left\{q^{j}\left[\begin{array}{c}
n-j \\
j
\end{array}\right]+\left[\begin{array}{c}
n-j-1 \\
j-1
\end{array}\right]\right\} s^{j} x^{n-2 j} \\
& =\sum_{j=0}^{k} q^{\left(\begin{array}{c}
j \\
2
\end{array}\right)} \frac{[n]}{[n-j]}\left[\begin{array}{c}
n-j \\
j
\end{array}\right] s^{j} x^{n-2 j} \\
& =\mathscr{L}_{n}^{k}
\end{aligned}
$$

for $0 \leqq k \leqq \frac{n}{2}$.

Theorem 4. The following recurrence relation holds true:

$$
L_{n}^{k}(x, q s, q)=\mathscr{F}_{n+1}^{k}+q^{n}{ }_{s} \mathcal{F}_{n-1}^{k-1} \quad\left(0 \leqq k \leqq \frac{n}{2}\right) .
$$


Proof. We consider

$$
\begin{aligned}
& \mathcal{F}_{n+1}^{k}+q^{n}{ }_{s} \mathcal{F}_{n-1}^{k-1}=\sum_{j=0}^{k} q^{\left(\begin{array}{c}
j+1 \\
2
\end{array}\right)}\left[\begin{array}{c}
n-j \\
j
\end{array}\right] s^{j} x^{n-2 j} \\
& +q^{n} s \sum_{j=0}^{k-1} q^{\left(\begin{array}{c}
j+1 \\
2
\end{array}\right)}\left[\begin{array}{c}
n-j-2 \\
j
\end{array}\right] j s^{j} x^{n-2-2 j}
\end{aligned}
$$

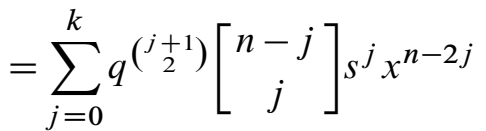

$$
\begin{aligned}
& +q^{n} \sum_{j=1}^{k} q^{\left(\begin{array}{l}
j \\
2
\end{array}\right)}\left[\begin{array}{c}
n-j-1 \\
j-1
\end{array}\right] s^{j} x^{n-2 j} \\
& =\sum_{j=1}^{k} q^{\left(\begin{array}{c}
j \\
2
\end{array}\right)}\left\{q^{j}\left[\begin{array}{c}
n-j \\
j
\end{array}\right]+q^{n}\left[\begin{array}{c}
n-j-1 \\
j-1
\end{array}\right]\right\} s^{j} x^{n-2 j} \\
& =\sum_{j=1}^{k} q^{\left(\begin{array}{l}
j \\
2
\end{array}\right)} q^{j} \frac{[n]}{[n-j]}\left[\begin{array}{c}
n-j \\
j
\end{array}\right] s^{j} x^{n-2 j} \\
& =L_{n}(x, q s, q)
\end{aligned}
$$

for $0 \leqq k \leqq \frac{n}{2}$.

Theorem 5. The recurrence relation of incomplete $q$-Lucas polynomials is given by

$$
\mathscr{L}_{n+2}^{k+1}=x \mathscr{L}_{n+1}^{k+1}+(q-1) s D_{q} \mathscr{L}_{n+1}^{k}+s \mathscr{L}_{n}^{k} \quad\left(0 \leqq k \leqq \frac{n-1}{2}\right) .
$$

Proof. In view of (2.3) and (2.5), we find that

$$
\begin{aligned}
& \mathscr{L}_{n+2}^{k+1}=\mathcal{F}_{n+3}^{k+1}+s \mathcal{F}_{n+1}^{k} \\
& =\left(x \mathcal{F}_{n+2}^{k+1}+(q-1) s D_{q} \mathscr{F}_{n+2}^{k}+s \mathscr{F}_{n+1}^{k}\right) \\
& +s\left(x \widetilde{F}_{n}^{k}+(q-1) s D_{q} \widetilde{F}_{n}^{k-1}+s \mathscr{F}_{n}^{k-1}\right) \\
& =x\left(\mathscr{F}_{n+2}^{k+1}+s \mathscr{F}_{n}^{k}\right)+(q-1) s D_{q}\left(\mathscr{F}_{n+2}^{k}+\mathscr{F}_{n}^{k-1}\right)+s\left(\mathscr{F}_{n+1}^{k}+s \mathscr{F}_{n-1}^{k-1}\right) \\
& \left.=x \mathscr{L}_{n+1}^{k+1}+(q-1) s D_{q} \mathscr{L}_{n+1}^{k}\right)+s \mathscr{L}_{n}^{k}
\end{aligned}
$$

for $0 \leqq k \leqq \frac{n-1}{2}$. 
Theorem 6. The following non-homogeneous recurrence relation of the incomplete q-Lucas polynomials holds true:

$$
\begin{aligned}
\mathscr{L}_{n+2}^{k}= & \left(x+(q-1) s D_{q}\right) \mathscr{L}_{n+1}^{k} \\
& +s \mathscr{L}_{n}^{k}-\frac{q^{\left(\begin{array}{c}
k \\
2
\end{array}\right)} q^{n}\left(q^{-k}[n+1]-1\right)}{[n-k]}\left[\begin{array}{c}
n-k \\
k
\end{array}\right] s^{k+1} x^{n-2 k} .
\end{aligned}
$$

Proof. It is easy to derive non-homogeneous recurrence relation (2.8) by using (2.2) and (2.7).

\subsection{Summation formulas}

Theorem 7. The following summation formula for the incomplete q-Fibonacci polynomials holds true:

$$
\sum_{j=0}^{h-1} \frac{1}{x^{j}}\left(s \mathcal{F}_{n+j}^{k}+(q-1) s D_{q} \mathscr{F}_{n+1+j}^{k}\right)=\frac{1}{x^{h-1}} \mathscr{F}_{n+1+h}^{k+1}-x \mathscr{F}_{n+1}^{k+1},
$$

where $x \neq 0$ and $n \geqq 2 k+2$.

Proof. Our proof uses the principle of mathematical induction on $h$. For $h=1$, the equation (2.9) holds true. Suppose that the equation (2.9) holds true for some integer $h>1$. Then, by using the equation (2.1), we get

$$
\begin{aligned}
& \sum_{j=0}^{h} \frac{1}{x^{j}}\left(s \widetilde{F}_{n+j}^{k}+(q-1) s D_{q} \mathscr{F}_{n+1+j}^{k}\right) \\
& =\sum_{j=0}^{h-1} \frac{1}{x^{j}}\left(s \mathcal{F}_{n+j}^{k}+(q-1) s D_{q} \widetilde{F}_{n+1+j}^{k}\right) \\
& +\frac{1}{x^{h}}\left(s \mathcal{F}_{n+h}^{k}+(q-1) s D_{q} \mathcal{F}_{n+1+h}^{k}\right) \\
& =\frac{1}{x^{h-1}} \mathscr{F}_{n+1+h}^{k+1}-x \mathcal{F}_{n+1}^{k+1}+\frac{1}{x^{h}}\left(s \mathcal{F}_{n+h}^{k}+(q-1) s D_{q} \mathscr{F}_{n+1+h}^{k}\right) \\
& =\frac{1}{x^{h}}\left(x \mathcal{F}_{n+1+h}^{k+1}+s \mathcal{F}_{n+h}^{k}+(q-1) s D_{q} \mathscr{F}_{n+1+h}^{k}\right)-x \mathcal{F}_{n+1}^{k+1} \\
& =\frac{1}{x^{h}} \mathcal{F}_{n+h+2}^{k+1}-x \mathcal{F}_{n+1}^{k+1},
\end{aligned}
$$

which completes the proof of the assertion (2.9) by the principle of mathematical induction on $h$. 
Theorem 8. A summation formula for the incomplete q-Lucas polynomials is given by

$$
\sum_{j=0}^{h-1} \frac{1}{x^{j}}\left(s \mathscr{L}_{n+j}^{k}+(q-1) s D_{q} \mathscr{L}_{n+1+j}^{k}\right)=\frac{1}{x^{h-1}} \mathscr{L}_{n+1+h}^{k+1}-x \mathscr{L}_{n+1}^{k+1},
$$

where $x \neq 0$ and $n \geqq 2 k+1$.

Proof. For using the principle of mathematical induction on $h$, we suppose that the assertion (2.10) is true for some integer $h>1$. We thus find from the equation (2.7) that

$$
\begin{aligned}
\sum_{j=0}^{h} \frac{1}{x^{j}}\left(s \mathscr{L}_{n+j}^{k}+(q-1) s D_{q} \mathscr{L}_{n+1+j}^{k}\right) \\
=\sum_{j=0}^{h-1} \frac{1}{x^{j}}\left(s \mathscr{L}_{n+j}^{k}+(q-1) s D_{q} \mathscr{L}_{n+1+j}^{k}\right) \\
\quad+\frac{1}{x^{h}}\left(s \mathscr{L}_{n+h}^{k}+(q-1) s D_{q} \mathscr{L}_{n+1+h}^{k}\right) \\
=\frac{1}{x^{h-1}} \mathscr{L}_{n+1+h}^{k+1}-x \mathscr{L}_{n}^{k+1}+\frac{1}{x^{h}}\left(s \mathscr{L}_{n+h}^{k}+(q-1) s D_{q} \mathscr{L}_{n+1+h}^{k}\right) \\
=\frac{1}{x^{h}}\left(x \mathscr{L}_{n+1+h}^{k+1}+s \mathscr{L}_{n+h}^{k}+(q-1) s D_{q} \mathscr{L}_{n+1+h}^{k}\right)-x \mathscr{L}_{n+1}^{k+1} \\
=\frac{1}{x^{h}} \mathscr{L}_{n+h+2}^{k+1}-x \mathscr{L}_{n+1}^{k+1},
\end{aligned}
$$

which completes the proof of the assertion (2.10) by the principle of mathematical induction on $h$.

We now derive some summation formulas for the incomplete $q$-Lucas polynomials.

Lemma 1. It is asserted that

$$
\sum_{j=0}^{\left\lfloor\frac{n}{2}\right\rfloor} q^{\left(\begin{array}{l}
j \\
2
\end{array}\right)} \frac{[n]}{[n-j]}\left[\begin{array}{c}
n-j \\
j
\end{array}\right] j s^{j} x^{n-2 j}=\frac{1}{2}\left(n \mathscr{L}_{n}-x \frac{d}{d x} \mathscr{L}_{n}\right) .
$$

Proof. We observe that

$$
\frac{d}{d x} \mathscr{L}_{n}=\sum_{j=0}^{\left\lfloor\frac{n}{2}\right\rfloor} q^{\left(\begin{array}{c}
j \\
2
\end{array}\right)} \frac{[n]}{[n-j]}\left[\begin{array}{c}
n-j \\
j
\end{array}\right] s^{j}(n-2 j) x^{n-2 j-1}
$$




$$
\begin{aligned}
= & n \sum_{j=0}^{\left\lfloor\frac{n}{2}\right\rfloor} q^{\left(\begin{array}{l}
j \\
2
\end{array}\right)} \frac{[n]}{[n-j]}\left[\begin{array}{c}
n-j \\
j
\end{array}\right] s^{j} x^{n-2 j-1} \\
& -2 \sum_{k=0}^{\left\lfloor\frac{n}{2}\right\rfloor} q^{\left(\frac{j}{2}\right)} \frac{[n]}{[n-j]}\left[\begin{array}{c}
n-j \\
j
\end{array}\right] j s^{j} x^{n-2 j-1} \\
= & n x^{-1} \mathscr{L}_{n}-2 x^{-1} \sum_{k=0}^{\left\lfloor\frac{n}{2}\right\rfloor} q^{\left(\begin{array}{l}
j \\
2
\end{array}\right)} \frac{[n]}{[n-j]}\left[\begin{array}{c}
n-j \\
j
\end{array}\right] j s^{j} x^{n-2 j},
\end{aligned}
$$

which proves the assertion (2.11) of Lemma 1.

The following theorem asserts the summation formula of the incomplete $q$-Lucas polynomials.

Theorem 9. Let $\mathscr{L}_{n}^{k}$ be the $n$th incomplete $q$-Lucas polynomial. Then

$$
\sum_{k=0}^{\left\lfloor\frac{n}{2}\right\rfloor} \mathscr{L}_{n}^{k}=\left(\left\lfloor\frac{n}{2}\right\rfloor-\frac{n}{2}+1\right) \mathscr{L}_{n}+\frac{x}{2} \frac{d}{d x} \mathscr{L}_{n}
$$

Proof. By using Definition 2 of the incomplete $q$-Lucas polynomials, we get

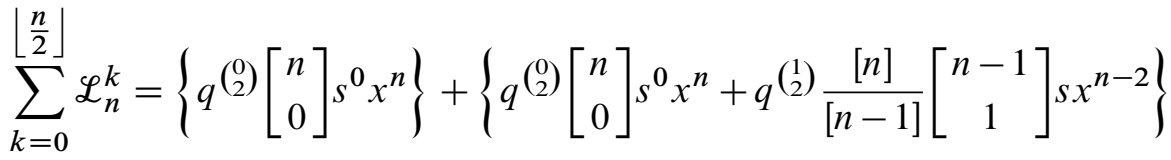

$$
\begin{aligned}
& +\left\{q^{\left(\begin{array}{l}
0 \\
2
\end{array}\right)}\left[\begin{array}{l}
n \\
0
\end{array}\right] s^{0} x^{n}+q^{\left(\begin{array}{l}
1 \\
2
\end{array}\right)} \frac{[n]}{[n-1]}\left[\begin{array}{c}
n-1 \\
1
\end{array}\right] s x^{n-2}+q^{\left(\frac{2}{2}\right)} \frac{[n]}{[n-2]}\left[\begin{array}{c}
n-2 \\
2
\end{array}\right] s^{2} x^{n-4}\right\}
\end{aligned}
$$

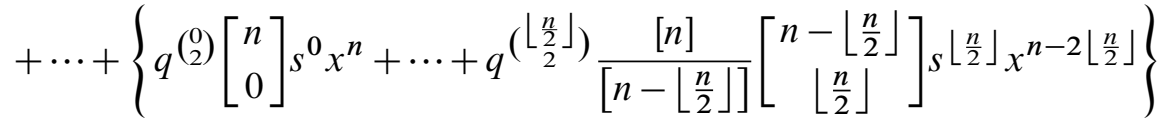

$$
\begin{aligned}
& =\sum_{k=0}^{\left\lfloor\frac{n}{2}\right\rfloor}\left(\left\lfloor\frac{n}{2}\right\rfloor+1-j\right) q^{\left(\frac{j}{2}\right)} \frac{[n]}{[n-j]}\left[\begin{array}{c}
n-j \\
j
\end{array}\right] s^{j} x^{n-2 j} \\
& =\sum_{k=0}^{\left\lfloor\frac{n}{2}\right\rfloor}\left(\left\lfloor\frac{n}{2}\right\rfloor+1\right) q^{\left(\begin{array}{l}
j \\
2
\end{array}\right)} \frac{[n]}{[n-j]}\left[\begin{array}{c}
n-j \\
j
\end{array}\right] s^{j} x^{n-2 j}-\sum_{k=0}^{\left\lfloor\frac{n}{2}\right\rfloor} q^{\left(\begin{array}{c}
j \\
2
\end{array}\right)} j \frac{[n]}{[n-j]}\left[\begin{array}{c}
n-j \\
j
\end{array}\right] s^{j} x^{n-2 j},
\end{aligned}
$$


which, in view of Lemma 1, yields

$$
\sum_{k=0}^{\left\lfloor\frac{n}{2}\right\rfloor} \mathscr{L}_{n}^{k}=\left(\left\lfloor\frac{n}{2}\right\rfloor-\frac{n}{2}+1\right) \mathscr{L}_{n}+\frac{x}{2} \frac{d}{d x} \mathscr{L}_{n} .
$$

\section{Generating FunCtions}

In this section, we obtain the generating functions of the incomplete $q$-Fibonacci and the $q$-Lucas polynomials.

Lemma 2 (see [17]). Let $\left\{s_{n}\right\}_{n=0}^{\infty}$ be a complex sequence satisfying the following non-homogeneous recurrence relation:

$$
s_{n}=x s_{n-1}+y s_{n-2}+r_{n} \quad(n>p),
$$

where $\left\{r_{n}\right\}$ is a given complex sequence. Then the generating function $S^{k}(x, y ; t)$ of the sequence $\left\{s_{n}\right\}$ is given by

$$
S^{k}(x, y ; t)=\left(s_{0}-r_{0}+\left(s_{1}-x s_{0}-r_{0}\right) t+G(t)\right)\left(1-x t-y t^{2}\right)^{-1},
$$

where $G(t)$ denotes the generating function of the given sequence $\left\{r_{n}\right\}$.

Theorem 10. The generating function of the incomplete $q$-Fibonacci polynomials is given by

$$
\begin{aligned}
U^{k}(x, s, q ; t)= & t^{2 k+1}\left(\mathcal{F}_{2 k+1}+\left(\mathcal{F}_{2 k+2}-\left(x+(q-1) s D_{q}\right) \mathcal{F}_{2 k+1}\right) t\right. \\
& \left.+q^{\left({ }^{k+2}\right)} s^{k+1} t^{2} \frac{1}{(1-x t q)_{q}^{k+1}}\right)\left(1-\left(x+(q-1) s D_{q}\right) t-s t^{2}\right)^{-1}
\end{aligned}
$$

where $\widetilde{F}_{k}=F_{k}(x, s, q)$ are the q-Fibonacci polynomials.

Proof. From (2.1) and (2.4), we get

$$
\widetilde{F}_{n}^{k}=0 \quad(0 \leqq n \leqq 2 k+1)
$$

and

$$
\mathcal{F}_{2 k+1}^{k}=\mathscr{F}_{2 k+1} \quad \text { and } \quad \mathscr{F}_{2 k+2}^{k}=\mathscr{F}_{2 k+2} .
$$

Also, for $n \geqq 2 k+3$, we have

$$
\begin{aligned}
\mathscr{F}_{n+2 k+1}^{k}= & \left(x+(q-1) s D_{q}\right) \mathscr{F}_{n+2 k}^{k} \\
& +s \mathcal{F}_{n+2 k-1}^{k}-q^{\left(\begin{array}{l}
k \\
2
\end{array}\right)} q^{n+2 k-1}\left[\begin{array}{c}
n+k-2 \\
k
\end{array}\right] s^{k+1} x^{n-2} .
\end{aligned}
$$


Let

and

$$
s_{n}=\mathscr{F}_{n+2 k+1}^{k}
$$

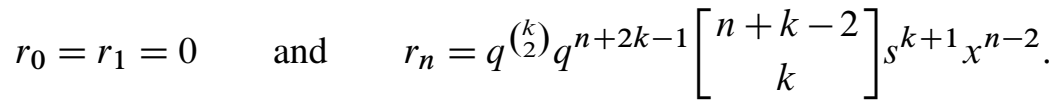

We then find that

$$
G(t)=q^{\left(\begin{array}{c}
k \\
2
\end{array}\right)} q^{2 k+1} s^{k+1} t^{2} \frac{1}{(1-x t q)_{q}^{k+1}}
$$

and

$$
\begin{aligned}
& S^{k}(x, s, q ; t)=\left(\mathscr{F}_{2 k+1}^{k}+\left(\mathscr{F}_{2 k+2}^{k}-\left(x+(q-1) s D_{q}\right) \mathscr{F}_{2 k+1}^{k}\right) t\right.
\end{aligned}
$$

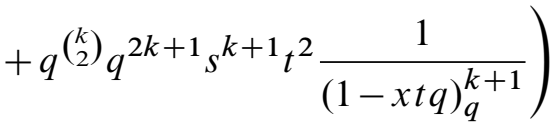

$$
\begin{aligned}
& \cdot\left(1-\left(x+(q-1) s D_{q}\right) t-s t^{2}\right)^{-1} \text {. }
\end{aligned}
$$

Therefore, we have

$$
U^{k}(x, s, q ; t)=t^{2 k+1} S^{k}(x, s, q ; t) .
$$

When $q \rightarrow 1-$ in Theorem 10, then we obtain the generating function of the incomplete bivariate Fibonacci polynomials (see [22]).

Theorem 11. The generating function of the incomplete q-Lucas polynomials is given by

$$
\begin{aligned}
& V^{k}(x, s, q ; t)=t^{2 k}\left(\mathscr{L}_{2 k}+\left(\mathscr{L}_{2 k+1}-\left(x+(q-1) s D_{q}\right) \mathscr{L}_{2 k}\right) t\right.
\end{aligned}
$$

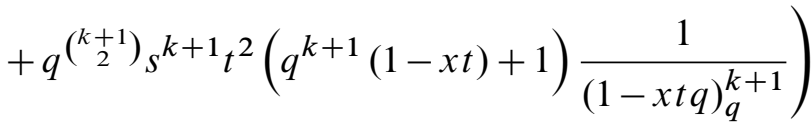

$$
\begin{aligned}
& \cdot\left(1-\left(x+(q-1) s D_{q}\right) t-s t^{2}\right)^{-1},
\end{aligned}
$$

where $\mathscr{L}_{k}=L_{k}(x, s, q)$ are the $q$-Lucas polynomials.

Proof. Considering the relation between the incomplete $q$-Fibonacci and $q$-Lucas polynomials, we get

$$
V^{k}(x, s, q ; t)=\sum_{n=0}^{\infty} \mathscr{L}_{n}^{k} t^{n}
$$




$$
\begin{aligned}
& =\sum_{n=0}^{\infty} \mathcal{F}_{n+1}^{k} t^{n}+s \sum_{n=0}^{\infty} \mathcal{F}_{n+1}^{k-1} t^{n} \\
& =t^{-1} U^{k}(x, s, q ; t)+s t U^{k-1}(x, s, q ; t) .
\end{aligned}
$$

\section{CONCLUSION}

In the present study, we have introduce the $q$-analogues of the incomplete Fibonacci and Lucas polynomials which satisfy essentially analogous recursion formulas and recurrence relations with the familiar $q$-Fibonacci and $q$-Lucas polynomials. Applications and some generalizations of the $q$-Fibonacci polynomials are given earlier in $[15,16]$, which contain some nice results for the $q$-Fibonacci polynomials. Also, Erkuş-Duman and Tuglu [12] studied various families of multilinear and multilateral generating functions for the generalized bivariate Fibonacci and Lucas polynomials. These works motivate the derivations of similar results for the incomplete $q$-Fibonacci and $q$-Lucas polynomials which we have investigated in this paper. By means of the relationships (1.8) and (1.9), we have exhibited the fact that the results for the $q$-analogues which we consider in this article for $0<q<1$ and the corresponding results for the $(p, q)$-analogues (with $0<q<p \leqq 1)$ are essentially equivalent, requiring only some obvious parametric variations, the additional parameter $p$ being redundant.

\section{REFERENCES}

[1] G. E. Andrews, "Fibonacci numbers and the Rogers-Ramanujan identities." Fibonacci Quart., vol. 42, no. 1, pp. 3-19, 2004.

[2] A. Aral, V. Gupta, and R. P. Agarwal, Applications of q-Calculus in Operator Theory. New York: Springer, 2013. doi: 10.1007/978-1-4614-6946-9.

[3] L. Carlitz, "Fibonacci notes. IV: q-Fibonacci polynomials." Fibonacci Q., vol. 13, no. 2, pp. 97102,1975

[4] J. Cigler, "Einige $q$-Analoga der Lucas-und Fibonacci-Polynome." Sitzungsber, Abt. II, Österr. Akad. Wiss. Math.-Naturwiss. Kl., vol. 211, pp. 3-20, 2002.

[5] J. Cigler, "A new class of $q$-Fibonacci polynomials." Electron. J. Combin., vol. 10, no. 3, pp. $1-15,2003$.

[6] J. Cigler, “q-Fibonacci polynomials.” Fibonacci Quart., vol. 41, no. 1, pp. 31-40, 2003.

[7] J. Cigler, " $q$-Fibonacci polynomials and the Rogers-Ramanujan identities." Ann. Combin., vol. 8, no. 3, pp. 269-285, 2004, doi: 10.1007/s00026-004-0220-8.

[8] G. B. Djordjević, "Generating functions of the incomplete generalized Fibonacci and generalized Lucas numbers." Fibonacci Quart., vol. 42, no. 2, pp. 106-113, 2004.

[9] G. B. Djordjević and H. M. Srivastava, "Incomplete generalized Jacobsthal and JacobsthalLucas numbers." Math. Comput. Model., vol. 42, no. 9-10, pp. 1049-1056, 2005, doi: 10.1016/j.mcm.2004.10.026.

[10] G. B. Djordjević and H. M. Srivastava, "Some generalizations of the incomplete Fibonacci and the incomplete Lucas polynomials." Adv. Stud. Contemp. Math. (Kyungshang), vol. 11, no. 1, pp. $11-32,2005$. 
[11] G. B. Djordjević and H. M. Srivastava, "Some generalizations of certain sequences associated with the Fibonacci numbers." J. Indones. Math. Soc., vol. 12, no. 1, pp. 99-112, 2006.

[12] E. Erkuş-Duman and N. Tuglu, "Generating functions for the generalized bivariate Fibonacci and Lucas polynomials." J. Comput. Anal. Appl., vol. 18, no. 5, pp. 815-821, 2005.

[13] P. Filipponi, "Incomplete Fibonacci and Lucas numbers." Rend. Circ. Mat. Palermo (2), vol. 45, no. 1, pp. 37-56, 1996, doi: 10.1007/BF02845088.

[14] C. Jia, H. M. Liu, and T. M. Wang, " $q$-Analogs of generalized Fibonacci and Lucas polynomials." Fibonacci Quart., vol. 45, no. 1, pp. 26-34, 2007.

[15] T. Mansour and M. Shattuck, "Free rises, restricted partitions, and $q$-Fibonacci polynomials." Afrika Mat., vol. 24, no. 3, pp. 305-320, 2013, doi: 10.1007/s13370-011-0060-8.

[16] E. Munarini, “Generalized $q$-Fibonacci numbers.” Fibonacci Quart., vol. 43, no. 3, pp. 234-242, 2005.

[17] A. Pintér and H. M. Srivastava, "Generating functions of the incomplete Fibonacci and Lucas numbers." Rend. Circ. Mat. Palermo (2), vol. 48, no. 3, pp. 591-596, 1999, doi: 10.1007/BF02844348.

[18] R. K. Raina and H. M. Srivastava, "A class of numbers associated with the Lucas numbers." Math. Comput. Model., vol. 25, no. 7, pp. 15-22, 1997, doi: 10.1016/S0895-7177(97)00045-9.

[19] J. L. Ramirez, "Incomplete generalized Fibonacci and Lucas polynomials." Hacet. J. Math. Statist., vol. 44, no. 2, pp. 363-373, 2015.

[20] H. M. Srivastava and J. Choi, Zeta and q-Zeta Functions and Associated Series and Integrals. Amsterdam: Elsevier, 2012.

[21] D. Tasci and M. Cetin-Firengiz, "Incomplete Fibonacci and Lucas p-numbers." Math. Comput. Model., vol. 52, no. 9-10, pp. 1763-1770, 2010, doi: 10.1016/j.mcm.2010.07.003.

[22] D. Tasci, M. Cetin-Firengiz, and N. Tuglu, "Incomplete bivariate Fibonacci and Lucas $p$ numbers." Discrete Dyn. Nature Soc., vol. 2012, Article ID 840345, pp. 1-11, 2012, doi: $10.1155 / 2012 / 840345$.

[23] J.-Z. Wang, "Some new results for the $(p, q)$-Fibonacci and Lucas polynomials." Adv. Difference Equ., vol. 2014, Article ID 64, pp. 1-15, 2014, doi: 10.1186/1687-1847-2014-64.

[24] W.-P. Wang and H. Wang, "Some results on convolved ( $p, q)$-Fibonacci polynomials." Integral Transforms Spec. Funct., vol. 26, no. 5, pp. 340-356, 2015, doi: 10.1080/10652469.2015.1007502.

\section{Authors' addresses}

\section{H. M. Srivastava}

Department of Mathematics and Statistics, University of Victoria, Victoria, British Columbia V8W 3R4, Canada, and Department of Medical Research, China Medical University Hospital, China Medical University, Taichung 40402, Taiwan, Republic of China

E-mail address: harimsriemath.uvic.ca

Naim Tuglu

Department of Mathematics, University of Gazi, TR-06500 Ankara, Turkey

E-mail address: naimtugluegazi.edu.tr

\section{Mirac Çetin}

Department of Mathematics Educations University of Başkent, TR-06810 Ankara, Turkey

E-mail address: mcetin@baskent.edu.tr 